\title{
Rebamipide upregulates mucin secretion of intestinal goblet cells via Akt phosphorylation
}

\author{
YURIKO YASUDA-ONOZAWA, OSAMU HANDA, YUJI NAITO, CHIHIRO USHIRODA, YOSUKE SUYAMA, \\ YUKI TOYOKAWA, TAKAAKI MURAKAMI, TOMOYO YASUDA, TOMOHIRO UEDA, ATSUSHI MAJIMA, \\ YUMA HOTTA, TOSHIFUMI DOI, MAKOTO TANAKA, YUSUKE HORII, YASUKI HIGASHIMURA, \\ KATSURA MIZUSHIMA, MAYUKO MORITA, YUKIKO UEHARA, HIDEKI HORIE, AKIFUMI FUKUI, \\ OSAMU DOHI, TETSUYA OKAYAMA, NAOHISA YOSHIDA, KAZUHIRO KAMADA, KAZUHIRO KATADA, \\ KAZUHIKO UCHIYAMA, TAKESHI ISHIKAWA, TOMOHISA TAKAGI, HIDEYUKI KONISHI and YOSHITO ITOH
}

Department of Molecular Gastroenterology and Hepatology, Kyoto Prefectural University of Medicine, Kyoto 602-8566, Japan

Received March 19, 2017; Accepted September 5, 2017

DOI: $10.3892 / \mathrm{mmr} .2017 .7647$

\begin{abstract}
Mucin is produced and secreted by epithelial goblet cells and is a key component of the innate immune system, acting as a barrier in the intestinal tract. However, no studies have been conducted investigating the increase in mucin secretion to enhance the intestinal barrier function. The present study investigated whether rebamipide (Reb) acts as a secretagogue of intestinal mucin and the underlying mechanisms involved, thereby focusing on the effect on goblet cells. The LS174T cell line was used as goblet cell-like cells. Using Reb-treated LS174T cells, the level of mucin content was assessed by periodic acid-Schiff (PAS) staining, and mucin 2, oligomeric mucus/gel-forming (MUC2) mRNA expression was assessed using quantitative polymerase chain reaction (PCR). Furthermore, MUC2 secretion in the supernatant was quantified by the dot blot method. The present study additionally investigated the involvement of the epidermal growth factor receptor/Akt serine/threonine kinase 1 (Akt) pathway in mucin secretion by western blotting. The results suggested that Reb strongly enhanced the positivity of PAS staining in LS174T cells, thereby suggesting increased intracellular mucin production. The PCR results indicated that Reb significantly increased MUC2 mRNA in whole cell lysate of LS174T cells. In order to assess the subsequent secretion of mucin by LS174T, MUC2 protein expression in the supernatant was assessed using the dot blot method and it was demonstrated that Reb significantly increased the secretion of MUC2 in a concentration-dependent manner. The p-Akt was significantly
\end{abstract}

Correspondence to: Dr Osamu Handa, Department of Molecular Gastroenterology and Hepatology, Kyoto Prefectural University of Medicine, 465 Kajii-cho, Kamigyo-ku, Kyoto 602-8566, Japan E-mail: handao@koto.kpu-m.ac.jp

Key words: rebamipide, mucin, goblet cell, intestine, Akt phosphorylation increased by Reb treatment, and an Akt inhibitor specifically suppressed MUC2 secretion. Overall, Reb increased mucin secretion directly via p-Akt. Reb-increased mucin may act as a strong non-specific barrier against pathogenic stimulants in various intestinal diseases.

\section{Introduction}

The mucus layer serves as the first line of defense in the intestine against pathogenic infections and mechanical injuries (1). The main component of mucus is mucin, which is synthesized and secreted by intestinal goblet cells (2). Intestinal mucin is a high molecular weight glycoprotein composed of O-linked glycosides connected with core proteins (3). The mucin core protein is called MUC, and more than 20 MUC types have been already identified (4). Among these MUC isoforms, mucin 2, oligomeric mucus/gel-forming (MUC2) is the main structural component of the mucus gel and represents the main secretory mucin in the small and large intestines $(5,6)$.

Various evidences have indicated the importance of intestinal mucin $(4,6,7)$ and its component MUC2 in maintaining intestinal homeostasis. The deficiency of mucin or MUC2 was also reported to be harmful as a result and/or a cause. In a mouse model, MUC2 deficiency led to the development of spontaneous colitis (3), and in humans, decrease in the number of mucin containing goblet cells was observed in patients with aspirin-induced intestinal injury (8), inflammatory bowel disease (9-11), and necrotizing enterocolitis (2). Therefore, maintenance or increase in intestinal mucin secretion is thought to be ideal for the prevention of or healing of many intestinal diseases. However, no drugs have been reported to increase mucin secretion in intestinal mucosa.

Rebamipide (Reb), a gastro muco-protective drug, has been used widely for more than 25 years and its safety has been confirmed $(12,13)$. Reb has been reported to increase mucin secretion in the conjunctiva (14) and stomach (15), but not in the intestine. However, the results of recent human studies have shown that Reb exerts protective effects against drug-induced intestinal injury (16-18). Reb can prevent diclofenac sodium-induced decrease of mucin in the small 
intestine (7); diclofenac sodium is a non-steroidal anti-inflammatory drug. Therefore, Reb can increase the secretion of intestinal mucin. In this study, we focused on goblet cells, which are mucin-secreting cell, to determine the possible use of Reb in increasing mucin secretion and the mechanism behind these phenomena.

\section{Materials and methods}

Cell culture and culture medium. LS174T [CL-188 ${ }^{\mathrm{TM}}$; American Type Culture Collection (ATCC); Manassas, VA, USA], a human colon adenocarcinoma cell line, exhibits characteristics of mucin-secreting intestinal epithelial cells and is widely used as an intestinal goblet cell $(6,19)$. LS174T were grown for 1 week in Eagle's minimum essential medium (EMEM) supplemented with $10 \%$ heat-inactivated fetal bovine serum (FBS), $100 \mathrm{U} / \mathrm{ml}$ penicillin, and $100 \mathrm{U} / \mathrm{ml}$ streptomycin. The cells were maintained at $37^{\circ} \mathrm{C}$ in a humidified incubator with $5 \% \mathrm{CO}_{2}$. LS174T were used in all experiments.

Prior to all experiments, the cells were serum-starved for $6 \mathrm{~h}$ in glucose-free EMEM. We seeded LS174T cells $\left(2 \times 10^{5}\right.$ cells $\left./ \mathrm{ml}\right)$ in 6 -well plates for protein assay and 24 -well plates for polymerase chain reaction (PCR) and dot blot assay.

Reagents. EMEM was purchased from ATCC and PBS and FBS were purchased from Invitrogen (Carlsbad, CA, USA). Reb was obtained from Otsuka Pharmaceutical Co., Ltd. (Tokyo, Japan). Epidermal growth factor (EGF; cat. no. E9644) was purchased from Sigma-Aldrich (St. Louis, MO, USA). Rabbit polyclonal anti-MUC2 (cat. no. NBP1-31231; 1:3,000 dilution) was purchased from Novus (St. Louis, MO, USA). Rabbit monoclonal anti-total EGF receptor (EGFR) (D38B1; cat. no. 6627; 1:1,000 dilution), rabbit monoclonal anti-phospho-EGFR Tyr ${ }^{1068}$ (cat. no. $3777 ; 1: 1,000$ dilution), rabbit monoclonal anti-total Akt (cat. no. 9272; 1:1,000 dilution), rabbit monoclonal anti-phospho-Akt Ser ${ }^{473}$ (cat. no. 4060; 1:2,000 dilution), and rabbit monoclonal anti-phospho-Akt $\mathrm{Thr}^{308}$ (cat. no. 4060; 1:2,000 dilution) were purchased from Cell Signaling Technology, Inc. (Danvers, MA, USA). Mouse monoclonal anti- $\beta$-actin antibody (cat. no. A5316; 1:1,000 dilution) was purchased from Sigma-Aldrich.

Cell viability. LS174T cells $\left(2 \times 10^{4}\right.$ cells $\left./ \mathrm{ml}\right)$ were grown in 96-well plates until confluence and incubated with Reb $(0,1$, 10 , and $100 \mu \mathrm{M}$ ) for $24 \mathrm{~h}$. Cell viability was quantified using a cell counting kit (Dojindo Laboratories, Tokyo, Japan) according to the manufacturer's instructions. After washing two times with PBS, the cells were incubated with methyl thiazolyl tetrazolium (MTT) solution for $2 \mathrm{~h}$ at $37^{\circ} \mathrm{C}$. The absorbance was measured at $450 \mathrm{~nm}$ using a microplate reader (SpectraMax M2; Molecular Devices, Sunnyvale, CA, USA). All experiments were performed in triplicate.

Periodic acid-Schiff (PAS) staining. LS174T cells $\left(2 \times 10^{4}\right.$ cells $\left./ \mathrm{ml}\right)$ were grown in 6-well plates until confluence and incubated with $\operatorname{Reb}(0,1,10$, and $100 \mu \mathrm{M})$ for $24 \mathrm{~h}$. Next LS174T cells were fixed in $10.5 \%$ formaldehyde at $4^{\circ} \mathrm{C}$ and stained using a PAS kit (Muto Pure Chemicals Co., Tokyo, Japan), according to the manufacturer's instructions.
Real-time PCR assay. Expression of MUC2, MUC5AC, and GAPDH mRNA in LS174T cells were determined using real-time PCR. Total RNA was isolated from LS174T cells using an RNA isolation reagent, Isogen (Nippon Gene Co., Ltd., Tokyo, Japan). Extracted RNA (1 mg) was reverse-transcribed into first-strand complementary DNA (cDNA) using the High-capacity cDNA Reverse Transcription kit (Applied Biosystems, Foster City, CA, USA). PCR reactions for MUC2, MUC5AC, and GAPDH were performed with the 7300 Real-time PCR system (Applied Biosystems) using the DNA-binding dye SYBR-Green to detect PCR products. The primers were of the following sequences: MUC2 sense, 5'-TGGGTGTCCTCGTCTCCTACA-3' and antisense, 5'-TGTTGCCAAACCGGTGGTA-3'; MUC5AC sense, 5'-TGCACGAAGCCTATGATCACTT-3' and antisense, 5'-GGCGCTGACATGGTAGTGGTA-3'; and GAPDH sense, 5'-ACCACAGTCCATGCCATCACT-3' and antisense, 5'-CCA TCACGCCACAGTTTCC-3'. All experiments were performed in triplicate.

Dot blot analysis. Dot blot analysis was used to measure the mucin concentration in a cell culture supernatant. Briefly, LS174T cells were incubated with $10 \mu \mathrm{M}$ Reb for $24 \mathrm{~h}$ in a 24-well plate. The collected supernatant was centrifuged at $1,000 \mathrm{~g}$ for $20 \mathrm{~min}$ at $20^{\circ} \mathrm{C}$, and the cell pellet was removed. Each supernatant was applied to each slot in a Bio-Dot $\mathrm{SF}^{\circledR}$ apparatus and blotted onto a nitrocellulose membrane (0.45 $\mu \mathrm{m}$; both from Bio-Rad Laboratories, Inc., Berkeley, CA, USA) by aspiration. The blotted membrane was incubated with $1 \%$ bovine serum albumin (BSA)-Tris-buffered saline and incubated with anti-MUC2 antibody $(1: 3,000$; Novus) overnight. After washing three times with $0.05 \%$ Tween-20-Tris-buffered saline (TBST), the membrane was incubated with secondary antibody, goat anti-rabbit $\mathrm{IgG}$ $(\mathrm{H}+\mathrm{L}$ )-AP (Bio-Rad Laboratories, Inc.) for $2 \mathrm{~h}$. The protein bands were visualized by Immun-Blot Goat Anti-Rabbit IgG $(\mathrm{H}+\mathrm{L})$-AP Assay kit (Bio-Rad Laboratories, Inc.). The bands for MUC2 on the membrane were quantified using Image $\mathbf{J}$ software (National Institutes of Health, Bethesda, MD, USA).

Next, in order to confirm the involvement of EGFR/Akt pathway in mucin secretion, we used two inhibitors; EGFR kinase inhibitor (AG1478, $200 \mathrm{nM}$ ) and PI3 kinase/Akt inhibitor (wortmannin, $10 \mu \mathrm{M}$ ). These inhibitors were added to LS174T cells $30 \mathrm{~min}$ prior to $10 \mu \mathrm{M}$ Reb (after this experiment, we used $10 \mu \mathrm{M}$ concentration of Reb) addition and incubated for $24 \mathrm{~h}$. The densities of MUC2 in the supernatant was measured by dot blot method above-mentioned and the bands for MUC2 on the membrane were quantified using Image $\mathbf{J}$ software. All experiments were performed in triplicate.

Western blot analysis. At first, we assessed the important signaling pathway for mucin secretion, p-EGFR/p-Akt, after the addition of $10 \mu \mathrm{M}$ Reb by western blotting. Treatment with $10 \mu \mathrm{M}$ Reb for different time periods $(0,15,30,60,120$, and $240 \mathrm{~min}), \mathrm{LS} 174 \mathrm{~T}$ were immediately rinsed with ice-cold PBS two times, and the cell pellet was dissolved with lysis buffer (Cell Lytic M; Sigma-Aldrich). These lysates were collected and incubated for $1 \mathrm{~h}$ on ice. After centrifugation at $12,000 \mathrm{~g}$ for $15 \mathrm{~min}$ at $4^{\circ} \mathrm{C}$, the supernatants were extracted, and the protein concentration was determined using a Bio-Rad Protein Assay kit (Bio-Rad Laboratories, Inc.). Protein (10 $\mu \mathrm{g})$ from 


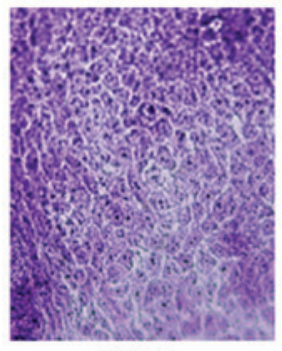

Control

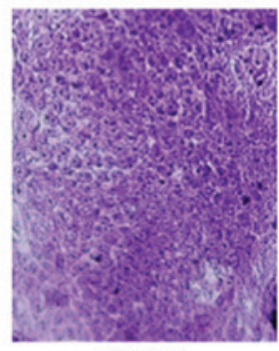

Reb $1 \mu \mathrm{M}$

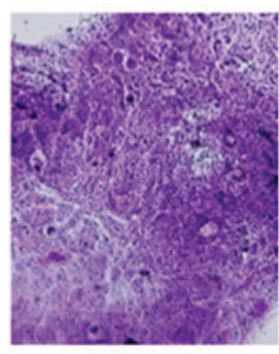

Reb $10 \mu \mathrm{M}$

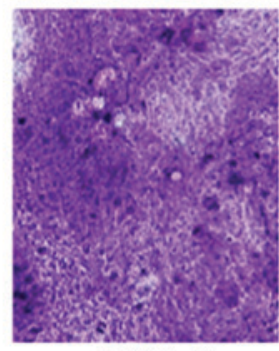

Reb $100 \mu \mathrm{M}$

Figure 1. Reb strongly upregulated mucin content in LS174T. Various concentrations of Reb (1-100 $\mu \mathrm{M})$ were added to LS174T for 24 h, and mucin production was assessed by PAS staining. Magnification, x40, Reb, rebamipide; PAS, periodic acid-Schiff.

each sample was electrophoresed on $10 \%$ SDS-PAGE gels for $30 \mathrm{~min}$ at $250 \mathrm{~V}$ and transferred to a nitrocellulose membrane (Invitrogen Japan K.K., Tokyo, Japan) using a semidry transfer system (Invitrogen Japan K.K.). The membrane was incubated for $1 \mathrm{~h}$ with a blocking solution (5\% BSA; Wako Pure Chemical Industries, Ltd., Osaka, Japan) in TBST (10 mM Tris. $\mathrm{Cl}$, pH 8.0, $150 \mathrm{mM} \mathrm{NaCl}$, and $0.1 \%$ Tween-20) at room temperature. After incubation with the appropriate primary antibody for $1 \mathrm{~h}$ at room temperature, the membrane was washed three times with TBST. The membrane was incubated in appropriate secondary antibody for $1 \mathrm{~h}$ at room temperature. Immunoreactive proteins were detected using a Western Blot Luminal Reagent kit (ECL plus; GE Health Bio-Sciences, Tokyo, Japan), and densitometry was measured using Image Quant TL software (GE Healthcare Life Sciences, Little Chalfont, UK).

During the next series of experiments, to confirm the active involvement of EGFR/Akt pathway in mucin secretion, we used three inhibitors; ERK1/2 kinase inhibitor (U0126, $1 \mu \mathrm{M}$ ), EGFR kinase inhibitor (AG1478, $200 \mathrm{nM}$ ), and PI3 kinase/Akt inhibitor (wortmannin, $10 \mu \mathrm{M})$. EGF (10 ng/ml, $15 \mathrm{~min}$ ) was used as the positive control to detect p-EGFR and p-Akt. Three inhibitors were added to LS174T cells 30 min prior to $10 \mu \mathrm{M}$ Reb addition and after 15 min LS174T whole cell lysates were collected. p-EGFR/p-Akt was detected by western blotting according to the method above-mentioned. All experiments were performed in triplicate.

\section{Results}

Reb upregulated mucin content in LS174T cell line. The Reb concentration $(1-100 \mu \mathrm{M})$ used in this study did not affect cell viability for $24 \mathrm{~h}$ (data not shown). After addition of Reb $(1-100 \mu \mathrm{M})$ to LS174T cells for $24 \mathrm{~h}$, the cells were stained using the PAS staining method. We found that Reb strongly upregulated the positivity of PAS staining in LS174T cells, regardless of the concentration (Fig. 1), thereby suggesting increased production of intracellular mucin. The Reb concentration $(10 \mu \mathrm{M})$ used in this study was thought to be a clinical relevant concentration, since the concentration of Reb in the human jejunum has been reported to be higher than $10 \mu \mathrm{M}$ at $3 \mathrm{~h}$ after oral intake of Reb (100 mg) (20), which is the dosage used in clinical practice.

Reb significantly increased MUC2 mRNA expression. To confirm the synthesis of MUC2 in Reb-treated LS174T cells,
A

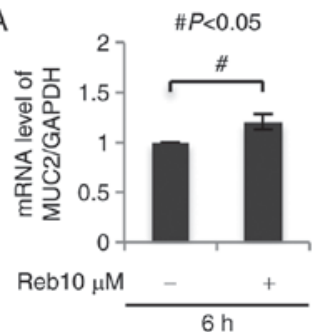

B

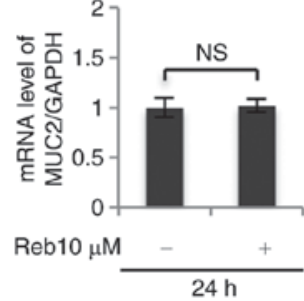

Figure 2. Reb significantly increased MUC2 mRNA expression. Reb $(10 \mu \mathrm{M})$ was added to LS174T, and the mRNA expressions of MUC2 were assessed by real time PCR. The graph shows mRNA level of MUC2/GAPDH $6 \mathrm{~h}$ (A) and $24 \mathrm{~h}$ (B) after Reb addition. Data were presented in mean $\pm \mathrm{SE}$ out of the three experiments. ${ }^{~} \mathrm{P}<0.05$. Reb, rebamipide; MUC2, mucin 2, oligomeric mucus/gel-forming; PCR, polymerase chain reaction; NS, not significant.
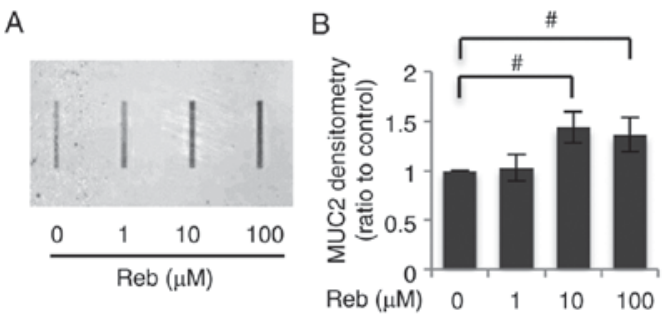

Figure 3. Reb significantly increased MUC2 secretion. Reb at various concentrations (1-100 $\mu \mathrm{M})$ was added to LS174T and MUC2 secretion in the culture supernatant was assessed by using dot blot method. (A) A representative image of the MUC2 bands was presented and (B) the graph of MUC2 densitometry data is shown. The mean density was quantified by Image $J$ software. Data were presented as mean \pm SE out of the three experiments. ${ }^{\#} \mathrm{P}<0.05$. Reb, rebamipide; MUC2, mucin 2, oligomeric mucus/gel-forming.

we assessed MUC2 expression by real time-PCR and found that Reb significantly increased MUC2 (intestinal mucin) mRNA expression after $6 \mathrm{~h}(\mathrm{P}=0.003$; Fig. 2$)$ without affecting MUC5AC (gastric mucin) mRNA expression (data not shown). These results suggest that Reb increased the synthesis of intestinal mucin, but not gastric mucin in LS174T cells.

Reb significantly increased MUC2 secretion. In order to assess the secretion of mucin by Reb-treated LS174T, we repeatedly assessed MUC2 protein secretion in the supernatant using western blotting and enzyme-linked immunosorbent assay (ELISA); however, we found it difficult to assess MUC2 secretion using these methods. There might be two possibilities for this difficulty; the western blotting for proteins with 
A
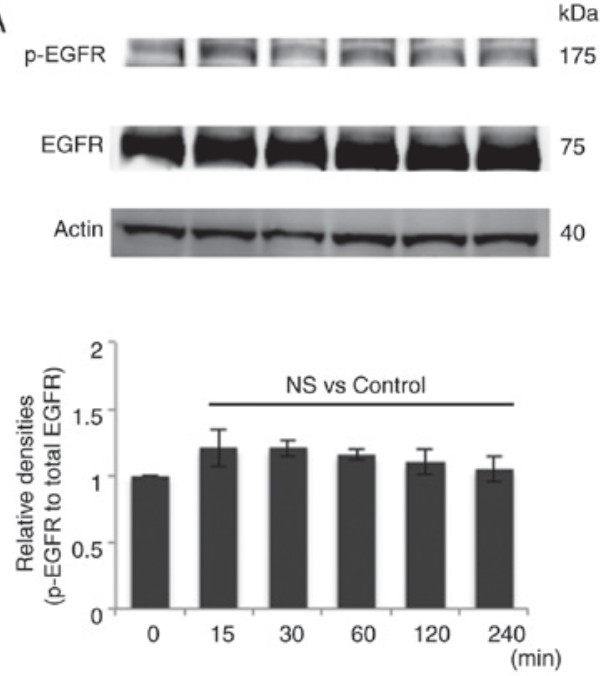

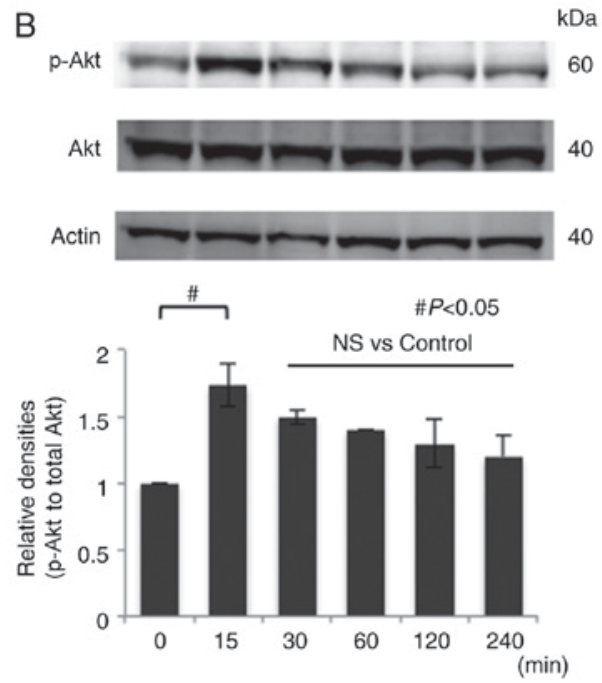

Figure 4. Reb significantly increased Akt phosphorylation after $15 \mathrm{~min}$. (A) Reb $(10 \mu \mathrm{M})$ was added to LS174T and the expression of EGFR and the p-EGFR at various periods were assessed by western blotting. Actin was used as an internal standard. A representative image was presented. The relative band density (p-EGFR/EGFR) was presented in the lower panel. Data were presented as mean \pm SE out of the three experiments; NS vs. the control. (B) Reb (10 $\mu \mathrm{M})$ was added to LS174T and the expression of Akt and the p-Akt at various periods were assessed by western blotting. Actin was used as the internal standard. A representative image was presented. The relative band density (p-Akt/Akt) was presented in the lower panel. Data were presented as mean \pm SE out of the three experiments; "P<0.05, NS vs. the control. Reb, rebamipide; EGFR, epidermal growth factor receptor; p-EGFR, phosphorylated EGFR; p-Akt, phosphorylated Akt; NS; not significant.
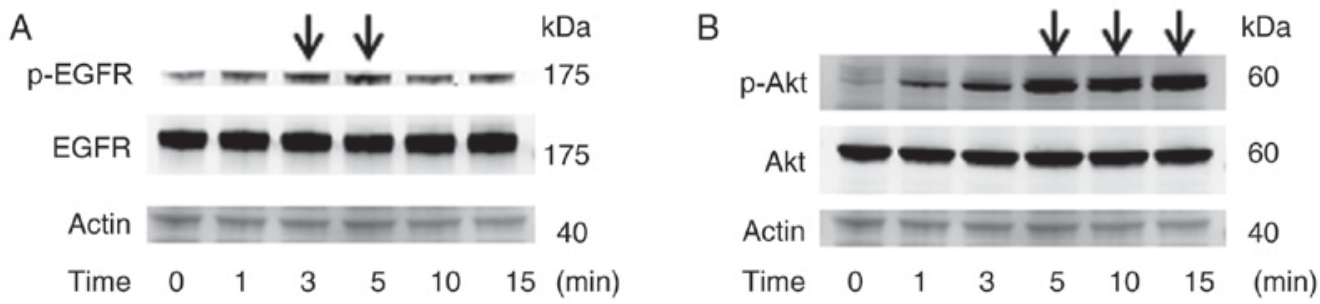

Figure 5. The peak of p-EGFR is shorter than p-Akt. EGF $(10 \mathrm{ng} / \mathrm{ml})$ was added to LS174T and the expression of EGFR/Akt and the phosphorylation of EGFR/Akt at various periods $(0,1,3,5,10$, and $15 \mathrm{~min})$ were assessed by western blotting. (A) The peak of phosphorylation of EGFR is 3-5 min after EGF addition, whereas (B) the peak of phosphorylation of Akt is 5-15 min after EGF addition. Actin was used as an internal standard (arrows indicate peaks). A representative image was presented. EGFR, epidermal growth factor receptor; p-EGFR, phosphorylated EGFR; EGF, epidermal growth factor; p-Akt, phosphorylated Akt.

very high molecular weight (MUC2, $520 \mathrm{kDa}$ ) might be difficult to perform or the amount of MUC2 secreted in the supernatant might be limited. To exclude the latter possibility, we employed a dot blot method and found that Reb increased MUC2 secretion in a concentration-dependent manner. Reb, at the concentration of more than $10 \mu \mathrm{M}$, significantly increased MUC2 secretion (Fig. 3).

Reb significantly increased $p$-Akt after 15 min. To elucidate the mechanism by which Reb increases mucin secretion, we treated LS174T cells with $10 \mu \mathrm{M}$ Reb for various periods $(0$, $15,30,60,120$, and $240 \mathrm{~min}$ ) and analyzed the phosphorylation status of EGFR and Akt, a well-known target of EGF, by western blotting. We found that Reb did not increase p-EGFR (Fig. 4A). On the contrary, Reb significantly increased p-Akt at serine 473 for 15 min (Fig. 4B). Since there are two phosphorylation sites of Akt, serine and threonine, we also examined phosphorylation at threonine 308 site, however Reb did not induce phosphorylation at this site (data not shown). We also examined phosphorylation of ERK1/2 after the treatment with $10 \mu \mathrm{M}$ Reb for different time periods $(0,15$,
30, 60, 120, and $240 \mathrm{~min}$ ) by western blotting; however, Reb did not induce p-ERK1/2 (data not shown). Taken together, we concluded that Reb induced phosphorylation at only serine 473 of Akt.

In our additional experiment, the maximum expression of p-EGFR or p-Akt was obtained 3-5 min or 5-15 min after EGF stimulation respectively (Fig. 5), suggesting that the signaling pathway of $\mathrm{p}$-EGFR is upstream of $\mathrm{p}$-Akt. Therefore, it is possible that Reb might induce maximum expression of p-EGFR at shorter than $15 \mathrm{~min}$, and we could not detect p-EGFR both in Reb and EGF stimulated LS174T cells at 15 min (Figs. 4 and 6).

Akt inhibitor suppressed Reb-induced p-Akt. To further evaluate the Reb-activated signaling pathway, we used three inhibitors; ERK1/2 kinase inhibitor (U0126, $1 \mu \mathrm{M}$ ), EGFR kinase inhibitor (AG1478, $200 \mathrm{nM}$ ), and PI3 kinase/Akt inhibitor (wortmannin, $10 \mu \mathrm{M}$ ). We found that Reb did not increase p-EGFR, and the three inhibitors did not affect p-EGFR (Fig. 6A). Among the three inhibitors, only wortmannin significantly suppressed Reb-increased p-Akt (Fig. 6B). Although 

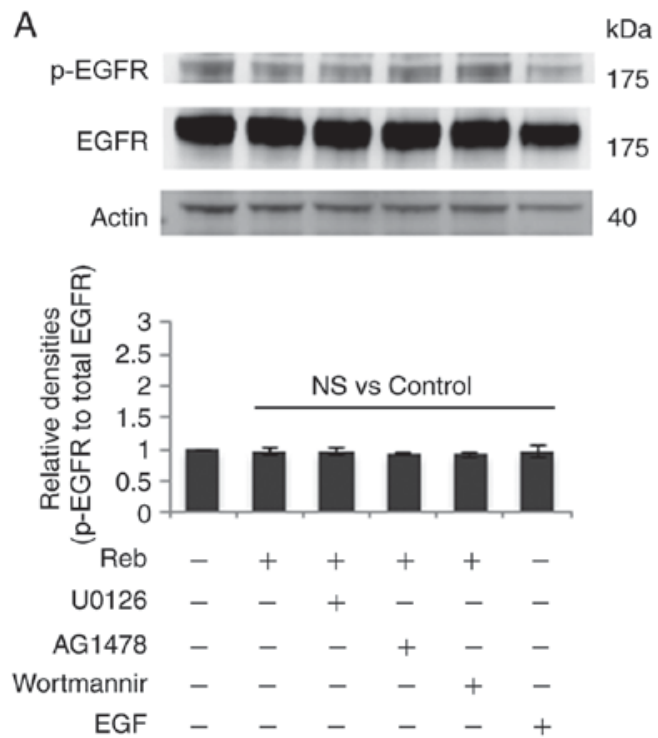

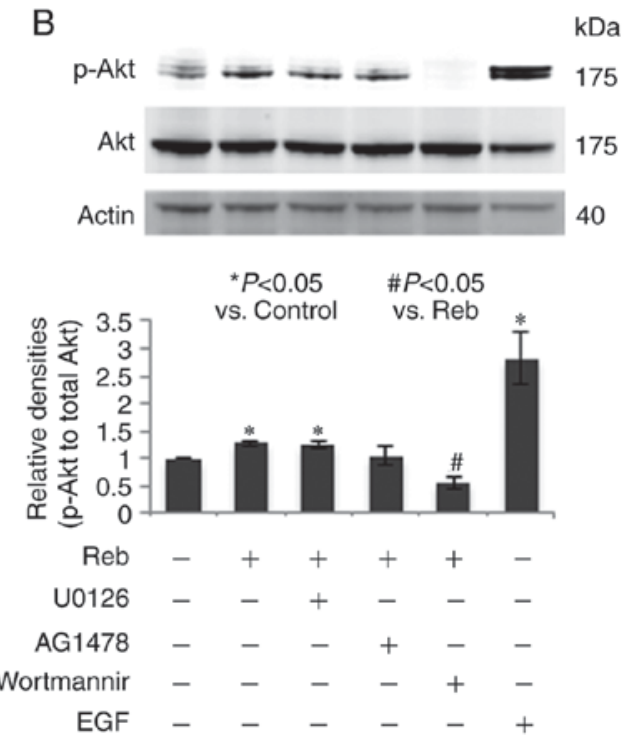

Figure 6. Akt inhibitor suppressed Reb-induced p-Akt. Reb $(10 \mu \mathrm{M})$ was added to LS174T cells and the expression of Akt and the p-Akt at 15 min were assessed by western blotting in the presence or absence of three inhibitors; ERK1/2 kinase inhibitor (U0126, $1 \mu \mathrm{M})$, EGFR kinase inhibitor (AG1478, 200 nM), and PI3 kinase/Akt inhibitor (wortmannin, $10 \mu \mathrm{M}$ ). Three inhibitors were added to LS174T cells 30 min prior to Reb addition. EGF (10 ng/ml, 15 min) was used as the positive control to detect (A) p-EGFR and (B) p-Akt. Actin was used as the internal standard. A representative image out of the three experiments was presented. The relative band density (p-EGFR/EGFR and p-Akt/Akt) was presented in the lower panel. Data were presented in mean \pm SE out of the three experiments. ${ }^{*} \mathrm{P}<0.05$ vs. the control; ${ }^{\#} \mathrm{P}<0.05$ vs. Reb, NS vs. the control. Reb, rebamipide; p-Akt, phosphorylated Akt; EGFR, epidermal growth factor receptor; EGF, epidermal growth factor; p-EGFR, phosphorylated EGFR; NS; not significant.

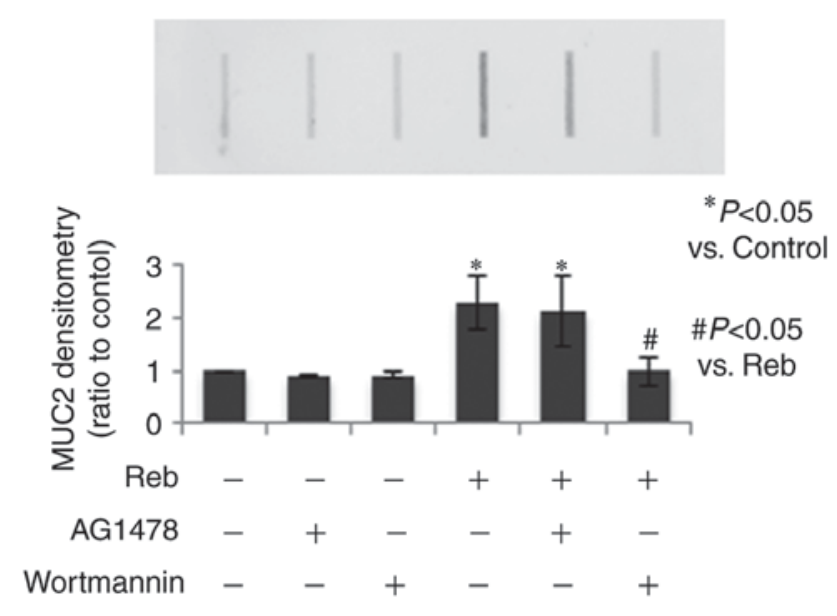

Figure 7. Reb significantly increased MUC2 secretion via p-Akt. Reb $(10 \mu \mathrm{M})$ was added to LS174T, and MUC2 secretion at $24 \mathrm{~h}$ was assessed by the dot blot method in the presence or absence of two inhibitors; EGFR kinase inhibitor (AG1478, $200 \mathrm{nM}$ ) and PI3 kinase/Akt inhibitor (wortmannin, $10 \mu \mathrm{M}$ ). Inhibitors were added to LS174T cells 30 min prior to Reb addition. A representative image out of the three experiments was presented. The band density was presented in the lower panel. Data were presented as mean $\pm \mathrm{SE}$ out of the three experiments. ${ }^{*} \mathrm{P}<0.05$ vs. the control; ${ }^{*} \mathrm{P}<0.05$ vs. Reb. Reb, rebamipide; MUC2, mucin 2, oligomeric mucus/gel-forming; p-Akt, phosphorylated Akt; EGFR, epidermal growth factor receptor.

MK-2206 would be a more specific inhibitor of Akt than wortmannin, the use of MK-2206 in LS174T cell has not been reported. Since several papers have reported the inhibitory effect of wortmannin in Akt signaling, we used wortmannin in this experiment.

Reb significantly increased MUC2 secretion via $p$-Akt. To confirm the involvement of p-Akt in Reb-induced mucin secretion, we used two inhibitors, EGFR kinase inhibitor (AG1478,
$200 \mathrm{nM}$ ) and PI3 kinase/Akt inhibitor (wortmannin, $10 \mu \mathrm{M}$ ) for the dot blot analysis. We found that Reb-increased MUC2 secretion was significantly reduced by wortmannin but not by AG1478 (Fig. 7), thereby suggesting that Reb specifically increased MUC2 secretion via p-Akt.

\section{Discussion}

In the present study, we found for the first time that a gastromucoprotective drug, Reb, increases mucin secretion in intestinal goblet cells. The most significant finding of the present study was that Reb strongly increased intracellular mucin production by PAS staining method and that the increase of MUC2 secretion in the cell culture supernatant was confirmed by dot blot analysis using a MUC2 specific antibody. Similar to previous studies that indicate Reb-increased mucin secretion in the conjunctiva (14) and stomach (15), Reb can increase mucin secretion by intestinal goblet cells, thereby suggesting that even in the intestine Reb can act as a prophylactic agent or a treatment drug for various intestinal diseases.

In this study, Reb significantly increased MUC2 mRNA expression in LS174T cells using real time PCR method; however, this did not lead to an upregulation in MUC2 protein expression as assessed by western blotting (data not shown). To confirm the Reb-induced MUC2 secretion in the cell culture supernatant, we performed ELISA; however, Reb-induced MUC2 secretion by LS174T was lower than the detection limit. Therefore, we used the dot blot method in order to detect small amounts of MUC2 protein; in this method all proteins in the cell culture supernatant are forced to bind directly to the membranes due to creation of a vacuum and MUC2 proteins can be detected by a specific antibody. This method revealed that Reb significantly increased MUC2 secretion. Taken together, we concluded that Reb increases MUC2 mRNA 
expression and synthesized MUC2 will be directly secreted into the cell culture supernatant.

In the next series of experiments, we investigated the mechanism by which Reb increases mucin secretion. It has been reported that Reb increases mucin secretion in the stomach $(15,21)$ and eyes $(22)$. However, only two studies have investigated the signal transduction mechanism by which Reb induces mucin secretion. In the human corneal epithelia, Reb upregulates MUC1, MUC4, and MUC16 expression via the activation of EGFR (23) and in rat conjunctival goblet cells, Reb induces mucin secretion by the activation of the EGFR and the MAPK pathway (14). In addition, in intestinal goblet cell-like cells, LS174T, EGF, and Lactobacillus-derived protein, $\mathrm{p} 40$, has been shown to enhance mucin secretion via trans-activation of EGFR/Akt pathway (1). Therefore, we assessed the phosphorylation status of EGFR and Akt after Reb treatment and found that Reb significantly upregulated p-Akt for 15 min without affecting p-EGFR. The difference between the results of our study and those of a previous study may be due to the difference in the methodology. However, almost all methodologies employed in our study, especially antibodies, are same as those used in the previous study. The antibodies (for EGFR, p-EGFR, Akt and p-Akt) were purchased from the same company and were used at the recommended concentration. The antibody for p-EGFR recognises only Tyr1068, therefore we also tried a different antibody to detect p-EGFR that recognises Tyr1148 (rabbit monoclonal anto-phophoEGFR Tyr1148, cat. no. 4404; 1:1,000 dilution); however, Reb did not upregulate p-EGFR as assessed by two different antibodies. The other possibility is that LS174T cells used in this study might be a different clone of LS174T. In some reports, the expression of EGFR is very low and the phosphorylation is hardly detected in LS174T $(24,25)$, thereby suggesting the heterogeneity of the LS174T cell line. In the LS174T cells used in this study, Reb specifically activated Akt signaling without affecting p-EGFR.

To confirm the active involvement of $\mathrm{p}$-Akt on Reb-increased mucin secretion, we used two inhibitors in dot blot analysis to evaluate MUC2 secretion: EGFR kinase inhibitor (AG1478) and PI3 kinase/Akt inhibitor (wortmannin). In this study, wortmannin, but not AG1478, significantly suppressed Reb-increased MUC2 secretion. This result strongly supported our hypothesis that Reb directly induces p-Akt bypassing the EGFR pathway; however, the mechanism through which Reb induces p-Akt is yet to be elucidated.

The limitation of this study is that we did not prove the protective effect of Reb-increased mucin on intestinal mucosal injury. Since we reported that aspirin-increased para-cellular permeability might be one of the mechanisms of aspirin-induced small intestinal mucosal injury (26), the in vitro experiment that tried to determine whether mucin secreted from goblet cells can suppress aspirin-induced increase of para-cellular permeability might clarify the protective effect of Reb. Animal experiments to elucidate whether Reb can increase mucin secretion in the intestine are also important.

The other limitation of this study is that the involvement of prostaglandin, which is reported to be a mucin secretagogue, was not investigated. Many studies have reported the importance of prostaglandin in promoting gastric epithelial mucin secretion in rat stomach $(27,28)$. However, only two studies have clearly stated the relationship between prostaglandin and intestinal mucin secretion in the intestine $(2,29)$. Moreover, McElroy et al (2) have shown that the addition of prostaglandin alone did not increase mucin secretion in rat experiments. However, this should be confirmed under experimental conditions in a future study.

Taken together, we concluded that Reb increased mucin secretion directly via p-Akt. Further, Reb-increased mucin could be a strong non-specific barrier against pathogenic stimulants in various intestinal diseases.

\section{Acknowledgements}

This study was supported by Grants-in-Aid for Scientific Research (KAKENHI) (C) to Y.N. (no. 25460958) from the Japan Society for the Promotion of Science (JSPS), and by an Adaptable and Seamless Technology Transfer Program through target driven R\&D (to Y.N.) from the Japan Agency for Medical Research and Development (AMED).

\section{References}

1. Wang L, Cao H, Liu L, Wang B, Walker WA, Acra SA and Yan F: Activation of epidermal growth factor receptor mediates mucin production stimulated by $\mathrm{p} 40$, a Lactobacillus rhamnosus GG-derived protein. J Biol Chem 289: 20234-20244, 2014.

2. McElroy SJ, Prince LS, Weitkamp JH, Reese J, Slaughter JC and Polk DB: Tumor necrosis factor receptor 1-dependent depletion of mucus in immature small intestine: A potential role in neonatal necrotizing enterocolitis. Am J Physiol Gastrointest Liver Physiol 301: G656-G666, 2011.

3. Iwashita J, Sato Y, Sugaya H, Takahashi N, Sasaki H and Abe T: mRNA of MUC2 is stimulated by IL-4, IL-13 or TNF-alpha through a mitogen-activated protein kinase pathway in human colon cancer cells. Immunol Cell Biol 81: 275-282, 2003.

4. Corfield AP: Mucins: A biologically relevant glycan barrier in mucosal protection. Biochim Biophys Acta 1850: 236-252, 2015.

5. Deplancke B and Gaskins HR: Microbial modulation of innate defense: Goblet cells and the intestinal mucus layer. Am J Clin Nutr 73: 1131S-1141S, 2001.

6. Elamin E, Masclee A, Troost F, Dekker J and Jonkers D: Cytotoxicity and metabolic stress induced by acetaldehyde in human intestinal LS174T goblet-like cells. Am J Physiol Gastrointest Liver Physiol 307: G286-G294, 2014.

7. Satoh H, Amagase $\mathrm{K}$ and Takeuchi K: Mucosal protective agents prevent exacerbation of NSAID-induced small intestinal lesions caused by antisecretory drugs in rats. J Pharmacol Exp Ther 348: 227-235, 2014.

8. Takeuchi T, Ensrud ER and Steggerda FR: The effects of large doses of aspirin and cortisone on the goblet cells and the mucosal membranes in the small and large intestine. Am J Dig Dis 17: 49-53, 1972.

9. Kim YS and Ho SB: Intestinal goblet cells and mucins in health and disease: Recent insights and progress. Curr Gastroenterol Rep 12: 319-330, 2010.

10. Jacobs LR and Huber PW: Regional distribution and alterations of lectin binding to colorectal mucin in mucosal biopsies from controls and subjects with inflammatory bowel diseases. J Clin Invest 75: 112-118, 1985.

11. Theodossi A, Spiegelhalter DJ, Jass J, Firth J, Dixon M, Leader M, Levison DA, Lindley R, Filipe I, Price A, et al: Observer variation and discriminatory value of biopsy features in inflammatory bowel disease. Gut 35: 961-968, 1994.

12. Naito Y and Yoshikawa T: Rebamipide: A gastrointestinal protective drug with pleiotropic activities. Expert Rev Gastroenterol Hepatol 4: 261-270, 2010.

13. Kim JH, Park SH, Cho CS, Lee ST, Yoo WH, Kim SK, Kang YM, Rew JS, Park YW, Lee SK, et al: Preventive efficacy and safety of rebamipide in nonsteroidal anti-inflammatory drug-induced mucosal toxicity. Gut Liver 8: 371-379, 2014. 
14. Ríos JD, Shatos MA, Urashima H and Dartt DA: Effect of OPC-12759 on EGF receptor activation, p44/p42 MAPK activity, and secretion in conjunctival goblet cells. Exp Eye Res 86: 629-636, 2008

15. Iijima K, Ichikawa T, Okada S, Ogawa M, Koike T, Ohara S and Shimosegawa T: Rebamipide, a cytoprotective drug, increases gastric mucus secretion in human: Evaluations with endoscopic gastrin test. Dig Dis Sci 54: 1500-1507, 2009.

16. Mizukami K, Murakami K, Abe T, Inoue K, Uchida M Okimoto T, Kodama M and Fujioka T: Aspirin-induced small bowel injuries and the preventive effect of rebamipide. World J Gastroenterol 17: 5117-5122, 2011.

17. Watanabe T, Takeuchi T, Handa O, Sakata Y, Tanigawa T, Shiba M, Naito Y, Higuchi K, Fujimoto K, Yoshikawa T and Arakawa T: A multicenter, randomized, double-blind, placebo-controlled trial of high-dose rebamipide treatment for low-dose aspirin-induced moderate-to-severe small intestinal damage. PLoS One 10: e0122330, 2015.

18. Lai Y, Zhong W, Yu T, Xia ZS, Li JY, Ouyang H, Shan TD, Yang HS and Chen QK: Rebamipide promotes the regeneration of aspirin-induced small-intestine mucosal injury through accumulation of $\beta$-catenin. PLoS One 10: e0132031, 2015.

19. van Klinken BJ, Oussoren E, Weenink JJ, Strous GJ, Büller HA, Dekker J and Einerhand AW: The human intestinal cell lines Caco-2 and LS174T as models to study cell-type specific mucin expression. Glycoconj J 13: 757-768, 1996.

20. Akamatsu T, Nagaya T, Ichikawa S, Sudo T, Takeda R, Takenaka K, Kodama R, Ito T, Arakura N and Tanaka E: Small bowel tissue concentration of rebamipide: Study of two dosages in healthy subjects. J Clin Biochem Nutr 47: 256-260, 2010.

21. Ishihara K, Komuro Y, Nishiyama N, Yamasaki K and Hotta K Effect of rebamipide on mucus secretion by endogenous prostaglandin-independent mechanism in rat gastric mucosa. Arzneimittelforschung 42: 1462-1466, 1992.
22. Urashima H, Okamoto T, Takeji Y, Shinohara H and Fujisawa S: Rebamipide increases the amount of mucin-like substances on the conjunctiva and cornea in the $\mathrm{N}$-acetylcysteine-treated in vivo model. Cornea 23: 613-619, 2004.

23. Itoh S, Itoh $\mathrm{K}$ and Shinohara H: Regulation of human corneal epithelial mucins by rebamipide. Curr Eye Res 39: 133-141, 2014.

24. Zhang X, Nagahara H, Mimori K, Inoue H, Sawada T, Ohira M, Hirakawa $\mathrm{K}$ and Mori M: Mutations of epidermal growth factor receptor in colon cancer indicate susceptibility or resistance to gefitinib. Oncol Rep 19: 1541-1544, 2008

25. Liu Z, Tabakman S, Sherlock S, Li X, Chen Z, Jiang K, Fan S and Dai $\mathrm{H}$ : Multiplexed five-color molecular imaging of cancer cells and tumor tissues with carbon nanotube raman tags in the near-infrared. Nano Res 3: 222-233, 2010.

26. Fukui A, Naito Y, Handa O, Kugai M, Tsuji T, Yoriki H, Qin Y, Adachi S, Higashimura Y, Mizushima K, et al: Acetyl salicylic acid induces damage to intestinal epithelial cells by oxidation-related modifications of ZO-1. Am J Physiol Gastrointest Liver Physiol 303: G927-G936, 2012.

27. Yamasaki K, Kanbe T, Chijiwa T, Ishiyama $H$ and Morita S: Gastric mucosal protection by OPC-12759, a novel antiulcer compound, in the rat. Eur J Pharmacol 142: 23-29, 1987.

28. Kleine A, Kluge S and Peskar BM: Stimulation of prostaglandin biosynthesis mediates gastroprotective effect of rebamipide in rats. Dig Dis Sci 38: 1441-1449, 1993.

29. Cassidy MM and Lightfoot FG: Effects of prostaglandin E1, administered by gastric intubation, on mucus secretory patterns in rat small intestine. Adv Prostaglandin Thromboxane Res 8: 1589-1593, 1980. 